\title{
Supervisory Control and Quality Assurance in Food Industry
}

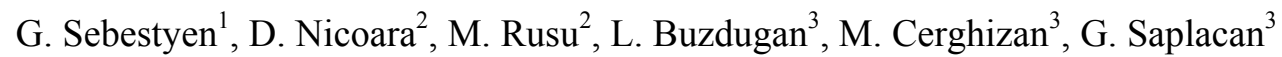 \\ ${ }^{1}$ Technical University of Cluj-Napoca, PhD, associated professor, gheorghe.sebestyen@cs.utcluj.ro \\ ${ }^{2}$ Company for Applied Informatics SA, office@cianet.ro \\ ${ }^{3}$ SC Sistec SA Cluj-Napoca
}

\begin{abstract}
The present paper describes the results of an interdisciplinary research project that combines knowledge from the domains of food processing, quality assurance, business modeling and ITC technologies in order to offer a general traceability solution for the Romanian food industry. This research complies with European Union's agricultural regulations which impose that food products must be accompanied along the whole producerconsumer chain, by documents regarding their origin, processing mode and quality.

The project's main goal is to design an integrated traceability and quality assurance information system for a generic food processing unit. The proposed system gives a global perspective to other business actors such as primary producers, distributors, retailers, warehouses, etc. The traceability system covers the following main functionalities: food processing lines' monitoring and supervision, traceability and quality information generation and recording and business-to-business data exchange.

In order to assure scalability and flexibility a multi-layer architecture was adopted, with a data management layer, an intra/inter-business layer and a unified GUI layer. The system is based on a Service Oriented Architecture, uses Web Services for data exchange and combines other new ITC technologies such as: SOAP, XML, WSDL, UDDI, and BPEL.
\end{abstract}

\section{INTRODUCTION}

In a global economy, as customer demands for food safety are growing, a systematic methodology for food safety and traceability assurance is compulsory. In order to have real-time access to relevant information concerning the quality of food products during the whole chain from the producer to the final consumer a complex distributed monitoring information system is needed. Such a system must record and offer access to the relevant parameters of a product as follows: origin, organoleptic features, processing procedures and their parameters, preservation locations and conditions, entities and personnel involved in the product's chain, etc.

At international level there are several regulatory and legislation initiatives that are meant to establish the legal framework for the development of quality and traceability assurance systems for the food industry. The European Union through its standardization and legislation boards adopted some important documents in the field, such as: The White Charta of food safety, The White paper on the quality systems HACCP, ISO-9000, ISO-22000, Directive of Europe's Council: nr.178/2002 "General Food Law", Directive 2001/95/EC “General Product Safety Requirement. The compulsoriness of assuring the required information for traceability of food products [8][9] determined a significant research and development effort in which important academic and industry entities were involved. The guidelines for the development of such traceability systems were established through a number of research projects. For instance TRACE [22] is a five year project involving more than $50 \mathrm{Eu}-$ ropean institutions and organizations. The project aims to develop generic and sector-specific traceability systems for use in the food industry. In order to fulfill the safety, quality and traceability requirements, Romania as member of the European Union initiated a set of legislative actions [22][23] and funded a number of research projects.

In the field of automatic product identification new coding systems (e.g. EPC - Electronic Product Code, UPC Universal Products Code) based on bar code or RFID technologies were proposed [1][2][6]. The Auto-ID project developed at MIT [15] is an excellent example of product identification procedure for traceability purposes.

An automated monitoring and traceability system [3][6][13] must provide customers and different supervising authorities, relevant information concerning critical stages of the products' lifecycle. Two information streams could be identified: downstream and upstream at the level of each operator in the chain [4].

This paper presents the results of a national research project which set as its goal to offer a quality and traceability framework for the Romanian companies involved in the production and processing of food products. The proposed and implemented framework includes both the infrastructure for the monitoring of food manufacturing lines and the services necessary to capture, classify, store and access data regarding products' quality in real-time regime. The information regarding the products and processes, stored in a database, are accessible through Web Services to business partners and authorities responsible for food quality tracing.

\section{A QUALITY AND TRACEABILITY MODEL}

The model of the proposed quality and traceability system was established in accordance with the following functional requirements:

- the information system must cover the distributed nature of food chains

- the food products and sub-products must be globally identifiable

- the system must offer the means to trace a product during its entire lifetime 
- the model should allow data exchange concerning products, upstream and downstream, between the entities involved in the food chain

- relevant information concerning the quality and safety of products must be stored during the lifecycle of a product, and specified amounts of time after

- the system must offer multiple access interfaces for different user types: producers, distributors, control authorities and end-consumers.

- interfaces must be well specified and clearly defined based on user roles and permissions.

- in case of safety alerts the system should help to quickly identify the source of risk, the products and entities involved, in order to facilitate product recall procedures

- the system must acquire, process and generate documents in accordance with the national and international regulations and standards

In order to fulfill these requirements a multi-level, distributed model is proposed. The model is organized as a set of autonomous applications centered on every entity participating in a food chain; the applications exchange data through a specialized protocol stack in order to assure the traceability requirements upstream and downstream of the food chain [10][11][12].

A production entity application has an intra-business part and an inter-business part. The intra-business part solves the following problems:

- monitors process parameters at production-floor level,

- associates product identification data and actual processing parameters

- stores relevant information about products during the production process

- generates official documents concerning the origin and quality of products

- generates alerts and logs events in case of unsafe production conditions

- impose predefined, pattern-based production and workflow rules (e.g. order of processing phases, parameters' limits, etc.).

The actors for the intra-business part are: production operators, managers, sails agents while the actors for the interbusiness part are: suppliers, distributors, control authorities, end-consumers.

The inter-business part assures business-to-business interoperability between food chain entities. The data exchange is made using standard high-level communication technologies and methods: XML, SOAP, UDDI, WSDL and Web services. For the communication model a pear-to-pear solution was adopted. The logic layer proposes that the communication is made through a set of predefined SOAP messages $[17][18][20]$. The format and the content of these messages were defined in accordance with the food industry business requirements, concerning quality, safety and traceability.

For an external application the present quality and traceability system is seen as a set of published web services. These services may be discovered using the UDDI technology and assure interoperability between partners and IT systems.

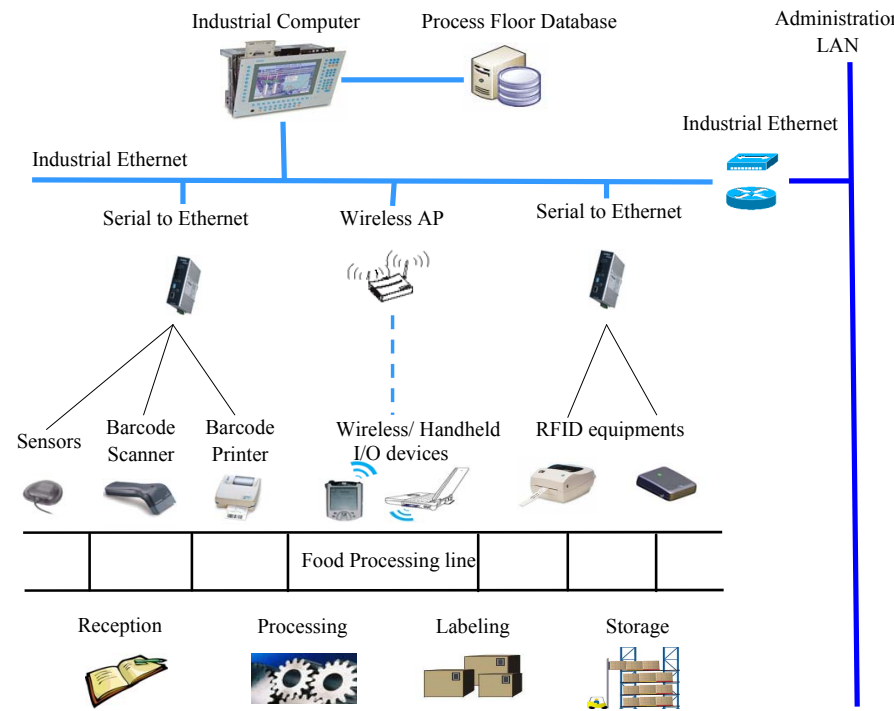

Figure 1. The physical scheme of the food process monitoring and supervision system

The data exchange is possible through the use of XML message formats.

\section{DATA ACQUISITION AND MONITORING}

The role of the monitoring module is to automatically acquire parameter values (e.g. temperature, humidity, gas composition, $\mathrm{pH}$, etc.) from critical measuring points placed along the food processing line. It also identifies the product units (sets, lots, piles, items) following the different phases of a processing workflow.

The monitoring module is composed of a set of distributed intelligent sensors, an industrial communication network and monitoring software (fig. 1). An important criterion in the selection of sensors was their ability to communicate through a serial channel or a network protocol. The solution with an industrial network is preferred because it assures a uniform access to all devices through a single cable and it offers the necessary reliability level imposed for industrial conditions. Unfortunately not all the necessary automation devices used for measuring purposes have a network interface or some are compliant with different protocols. Given these conditions the adopted solution was a mixed one:

- most of the sensors (e.g. temperature, humidity), LCD displays and alarm units were connected through a ModBusRS485, twisted-wire network

- devices with serial interface (RS-232) such as bare-code readers, RFID devices or weight measuring units were connected to an Ethernet network through a serial to Ethernet converter

- devices with wireless capabilities, such as hand-held devices (e.g. process-operator input/output devices) were linked to the system through a wireless access point

The ModBus protocol allows flexible access to the measured values and configuring parameters of the intelligent devices present in the network. Depending on the complexity of the food processing flow a variable number of devices can be connected on the network. The network software deployed in the 
process computer identifies the existing devices and through operator commands configures the functional conditions of devices (e.g. parameter limits, data acquisition frequency etc.).

The hand-held devices used by the process operators allow visualization of process data and manual input of some parameters that are not measured by devices with communication capabilities. The wireless interfaces of these devices offer mobility to operator through the entire process.

At logical level all the process-floor devices are modeled as virtual manufacturing devices (VMDs), with a unique access interface. In this way the higher level software components does not have to take in consideration all the particular characteristics of the different devices, including their communication capabilities (e.g. through a network, serial channel, wireless connection). Manually introduced data may be seen as a data coming from a virtual device. Behind the VMD concept there is a set of device drivers specialized for every kind of physical device. These drivers transmit the input/output data to the higher software levels through the same interface component.

Data acquisition consists of several threads with specific configurable - polling times. In the configuration phase the operator set the acquisition frequency and the allowed value interval for every process parameter. If a parameter exceeds the allowed interval an event is generated and the monitoring program treats it according to a predefined procedure.

The evolution of a product through the processing flow is monitored with the barcode reader or RFID devices placed in the critical measuring points of the flow. These devices determine the identity of the product set that leaves a given processing phase and enters in a new one. The monitoring software records the time of the transition and associates measured parameter values with product items. When a product set arrives at the end of the processing line all the parameter values measured in different phases are recorded on the documents associated with the final product. From the traceability point of view this automated data acquisition procedure guarantees that a product followed a given processing track and the recorded parameter values are real.

The system is based on a set of relational databases distributed at every entity involved in the food chain. At the production level the database contains around 100 tables with 3 types of data: configuration tables, catalogs and real-time monitoring tables. The configuration tables describe the domain ontology: the production unit, the production flows, the HACCP (Hazard Analysis Control Check Points) regulations and safety parameters limits [7].

The catalogs contain data about products, suppliers and distributors, measuring units and process parameters. The realtime monitoring tables collect data during the daily production processes. Here the products' identification data is associated with automatically measured workflow parameters. These data testifies the quality of product lots.

Every product lot is identified with a unique Electronic Product Code (EPC) [19][21][22]. A given actor (e.g. the endconsumer) may identify a product lot or item and ask for all the relevant information about the quality parameters of that product. The origin, the processing and storage conditions,

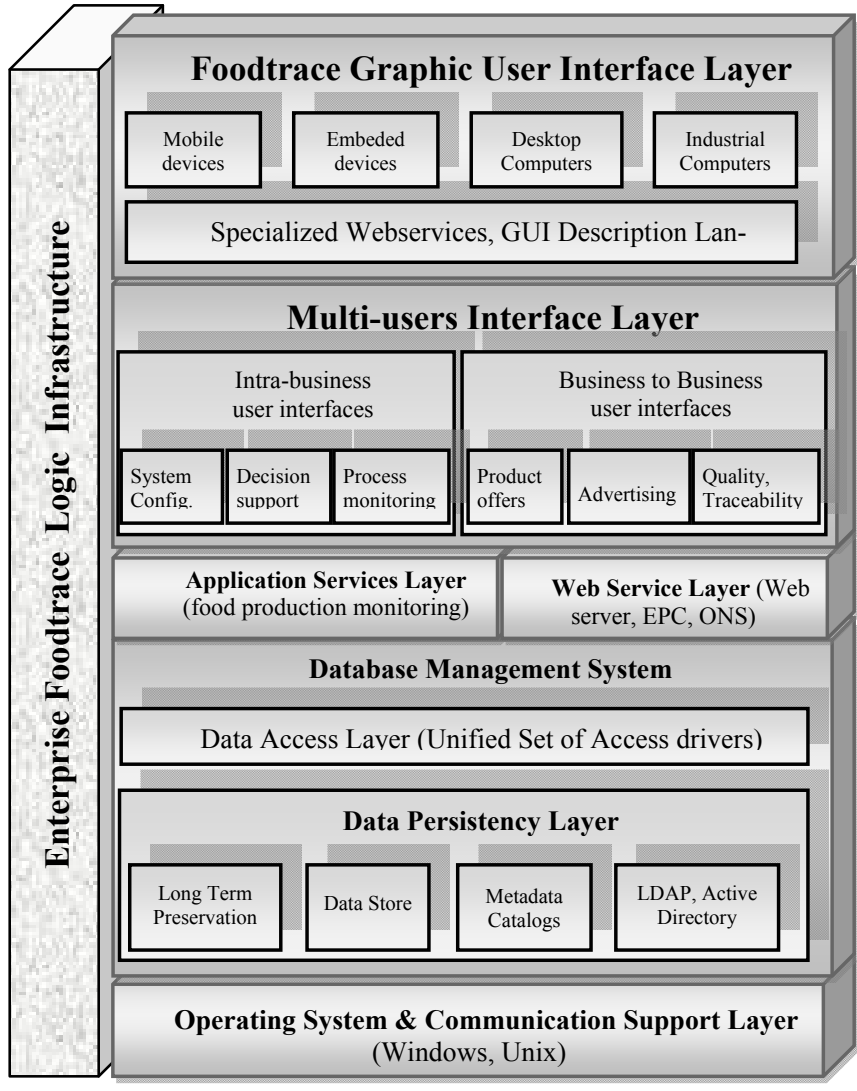

Figure 2. The Foodtrace Quality and Traceability System Multi-users Interface and GUI Layer

composition, etc. can be determined through the present system.

\section{DATA VisUALISATION}

Visualization and control are key features that assure the success of a traceability system. As important part of the system, the presentation layer proposed in the current framework is a highly extensible and configurable component providing a graphical access interface for real-time monitored parameters. It implements a unified presentation solution that handles both application and web portal intelligent controls. It uses a loosely-coupled command architecture with separate threads for GUI drawing and user generated events. The framework is designed to provide high interoperability, flexibility and ease development for third party developers.

Fig. 2 shows the logical multi-layered structure of the FoodTrace framework [14]. The visualization and control part is implemented through the GUI layer and the Multi-user Interface Layer. The Multi-user layer contains components for the intra-business users (for system configuration, decision support and process monitoring) and for the business-to-business data exchange (for product offers, advertising and traceability). This layer gives flexible access to the Application and web services layer. This part of the framework implements the process monitoring logic and the business rules of a company. All the information concerning products and their quality and safety characteristics are preserved and administered in the Database Management Layer. A unified set of access drivers and tem- 
plates facilitate the exchange of data between the upper layers and the actual database. Information concerning quality and traceability of food products are stored for a predefined period of time in accordance with regulations.

Different types of clients (thin, thick and portal clients) share a common GUI layer based on the extensible application markup language's (XAML) specifications [5]. The GUI description language used in the Foodtrace framework offers onthe-fly customization of design and behavior during and after deployment phase. In order to permit a high quality representation of the "intelligent maps" that visualize processes, vector graphics primitives inherited from XAML were combined with a set of vector-based GIS components; the solution is suitable for larger and more complex images that cannot be presented with raster methods.

Through this layer users with different roles and access rights can monitor food production lines, publish relevant information about products and their processing conditions, place and follow orders, get upstream and downstream information about products, generate and consult documents about the products' quality conformance, etc.

In order to access the resources of the Foodtrace system through SCADA enabled mobile devices, intelligent controls were developed. These controls assure cross-platform, crossarchitecture availability for data visualization and process control. A set of controls were developed for inline generation of graphics, plots and real-time alerts. The controls are linked to several data sources from the Data layer allowing both raw data visualization and graphics generation. They also offer the ability to control and change the production line parameters and/or recorded values. The generic datagrid controls were extended in order to add caching functionalities and all the raster images utilized in maps and plots are rendered on the server for faster application interaction. Secured web services that expose the information for the various graphic interfaces are designed to assure fast interaction and data manipulation; this is achieved through stored procedures, cached data and on-line interrogations.

The Foodtrace framework offers the possibility to use "intelligent maps" on SCADA enabled mobile devices for a precise localization, monitoring and control of sensors and equivalents logically mapped onto virtual devices. The notion of intelligent maps was used to denote controls capable of representing vector, raster and hybrid (vector+raster) maps allowing one user to easily trace the product's manufacturing components from raw materials to finite product. These maps allow supervisors to trace and identify problems on the production line, offering them the possibility to adjust parameters and take risk related decisions remotely. From the implementation point of view the maps can be accessed via specialized web services from any platform(Java/.NET).

The Foodtrace framework provides a stable SOA eventdriven platform for reliable exchange of messages and notifications, from data acquisition level up to user interface layer. Partners and entities involved in traceability can be notified about the occurrence of business events in the food chain.

All these functionalities may be accessed through the pre-

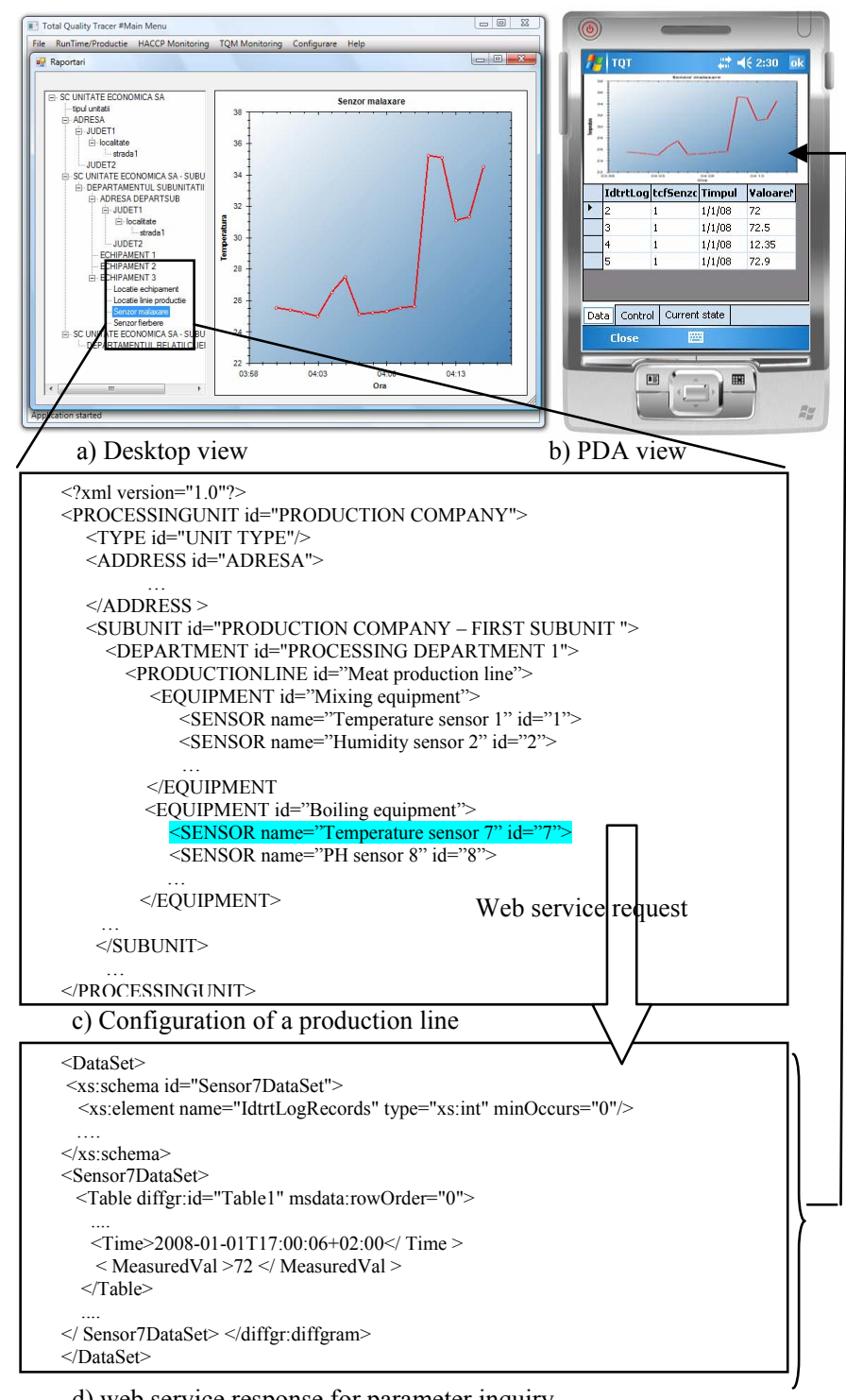

d) web service response for parameter inquiry

Figure 3. Foodtrace - The unified solution for desktop computers and PDAs

sented user interface layer by using any type of equipment: mobile phones, desktop computers, industrial computers.

The common user interface layer aggregates multiple channels of information and communication through a single interface, providing consistent design and functionality while hiding the differences between data-resources of the Foodtrace portal.

\section{IMPLEMENTATION ASPECTS}

The system was developed compliant with Service Oriented Architecture (SOA) and uses web-services based on several widely accepted standards (SOAP, XML, BPEL4WS, UDDI, WSDL). The system has intra-business traceability components for enterprise level supervision (Business-to-Enterprise) and also inter-business traceability components (Business-toBusiness) for business partners and global visibility.

In the deployment phase the system can be configured in accordance with the physical resources of different industrial workflows. The configuration modules generate and update a 
set of tables in the system's database (e.g. for sensors, unit structures, control points, technological operations, etc.). Changes in the production lines during exploitation can be easily updated in the system.

The FoodTrace framework assures import and export functionalities to other applications, without operator intervention, through XSLT transformations.

Fig. 3 shows operator interfaces on desktop and mobile devices (e.g. PDAs), that assure process supervision, monitoring and control. These interfaces offer a hierarchical view on the production unit infrastructure and process parameters. XML documents describe the production flow resources (Fig. 3 c) and the structure of the data messages (Fig. 3 d). Fig. $3 \mathrm{a}$ and $\mathrm{b}$ shows a sensor (e.g. Temperature sensor) as part of a configuration document, as a measured parameter value and as a graphical representation of its evolution.

In its actual phase the Foodtrace framework is adapted mainly for the meat processing lines, but it can be extended to other kind of food processing units.

\section{CONCLUSIONS}

This paper presents the main steps in the design and implementation of a complex distributed information system meant to monitor the quality of food products along their lifetime. The first part of the paper presents the functional and regulatory requirements imposed for a traceability and quality assurance system. Than a multi-level intra and inter-business system model is introduced. An important part of the paper deals with the automatic real-time monitoring of food processing lines. The database structure proposed in the paper covers all the aspects concerning products identification and process parameters recording during the lifetime of food products.

Different actors and entities involved in the food chain may access relevant information about products through specialized interfaces. Using standards and highly accepted data exchange technologies the proposed system has an open, flexible and scalable structure; it assures the interoperability with other enterprise applications.

\section{ACKNOWLEDGMENT}

The system presented in this paper was designed and developed in a national research project funded by the Romanian Ministry of education and research.

\section{REFERENCES}

[1] K. Goyal, D. Krishna, "RFID Middleware - Integration To The Entire Supply Chain", RFID Journal, 2005

[2] G. Capone, D. Costlow, S. Grenoble, R. Novack, "The RFID-Enabled Warehouse", Center for Supply-Chain Research, Penn State University, 2004

[3] M.G. Cimino, B. Lazzerini, F. Marcelloni, A. Tomasi, "Cerere: an information system supporting traceability in the food supply chain", Proceedings of the 7th International Conference on E-Commerce Technology Workshops (CECW'05), 2005

[4] S. Nedevschi, I. Salomie, G. Sebestyen., G. Saplacan, "Quality and traceability management system in Food industry", $5^{\text {th }}$ Interantional Congress of Food Technology, Thessaloniki, 2007, pp. 434-442

[5] L.A. MacVittie, "XAML in a Nutshell", First edition, O'Reilly, 2006

[6] M. Kerluk, "Track and trace labeling - the future chanllenge", Food Solutions Europe, http://www.foodsolutions.eu.com/pastissue/, 2008
[7] R.W. Daniels, "Applying HACCP to New-Generation Refrigerated Foods at Retail and Beyond", Food Technology 6, p. 112

[8] P. Shears, E.Z. Frances, N.H. Sandra, "The European Food Safety Authority: towards coherence in food safety policy and practice", British Food Journal, 2004, pp. 336-352

[9] C. Ansell, D. Vogel, "The contested Governance of European Food Safety", Institute of Governmental Studies, University of California Berkeley, 2005

[10] I. Rapanotti, J.G. Hall, M. Jackson, B. Nuseibeh, "Architecture-driven problem decomposition",Requirements Engineering Conference 2004. Proceedings. 12th IEEE International(2004), 2004, pp. 80-89

[11] E.V. Munson, N. Tien, "Concordance, conformance, versions, and traceability", 2005, pp. 62-66

[12] J. Bayer, T. Widen, "Introducing Traceability to Product Lines", 2002, pp. $409-416$

[13] G. Romanski, "Requirements, Configuration Management and Traceability for Safety Critical Software", 2003

[14] T. Takeno, A. Okamoto, M. Horikawa, T. Uetake, M. Sugawara, "ThreeLayered Traceability System Supporting both Blanching and Assembly Process", ICE Conference Proceedings, http://www.ejov.org/apps/comm.asp?Q $=408,2005$

[15] ***, "Auto-ID Project", http://autoid.mit.edu/web/, Massachusetts Institute of Technology

[16] ***, "Tracing the origin of food project", www.trace.eu.org/menu/project

[17] ***, "The OMG of Service Oriented Architecture", www.omg.org/attachments/pdf/OMG-and-the-SOA.pdf

[18] ***, "BPEL4WS Specifications", Business Process Modeling Notation V1, www.bpmn.org/Documents/BPMN, 2004

[19] ***, "EPCglobal Network", http://www.epcglobalinc.org/home

[20] ***, "Microsoft Biztalk Server", www.microsoft.com/biztalk/default.mspx, 2006

[21] ***, "Auto-ID Center - PML Core Specification", http://develop.autoidcenter.org

[22] ***, Ordinul 207/2007 al presedintelui Autoritatii Nationale Sanitar Veterinare si pentru Siguranta Alimentelor, Monitorul Oficial nr.4, 3 Ianuarie 2008

[23] ***, "Norme de igiena a produselor alimentare", HG 1198/2002 